\title{
COMPARAÇÃO ENTRE OS MÉTODOS DE AMOSTRAGEM DE BITTERLICH E DE ÁREA FIXA COM PARCELA CIRCULAR EM PLANTAÇÃO DE Pinus taeda
}

\author{
João Paulo Druszcz ${ }^{1}$, Nelson Yoshihiro Nakajima ${ }^{2}$, Sylvio Péllico Netto ${ }^{3}$, Mauro Yoshitani Júnior ${ }^{4}$ \\ ${ }^{1}$ Eng. Florestal, M.Sc., Silviconsult Engenharia, Curitiba, PR, Brasil - jpaulorz@ yahoo.com.br \\ ${ }^{2}$ Eng. Florestal, Dr., Depto. de Ciências Florestais, UFPR, Curitiba, PR, Brasil - nelson.nakajima@ufpr.br \\ ${ }^{3}$ Eng. Florestal, Dr., PUCPR, São José dos Pinhais, PR, Brasil - pellico.sylvio@ pucpr.br \\ ${ }^{4}$ Eng. Florestal, M.Sc., Curitiba, PR, Brasil - mauroyoshitani@yahoo.com.br \\ Recebido para publicação: 19/11/2007 - Aceito para publicação: 09/10/2009
}

\begin{abstract}
Resumo
Este estudo objetivou comparar o método de amostragem de Bitterlich e o de área fixa com Parcela Circular. Estes dois métodos foram avaliados quanto à precisão e eficiência na estimativa das variáveis DAP médio, número de árvores, área basal e volume total, por hectare. Foram selecionadas três condições de plantios de Pinus taeda, localizados na fazenda Boa Vista, no município de Carambeí - PR. Na análise simultânea das três condições para as estimativas das variáveis DAP médio e número de árvores, por hectare, o método de amostragem de área fixa com Parcela Circular foi o mais preciso e eficiente. Já para a análise de área basal e volume total, por hectare, o método de amostragem de Bitterlich foi o mais preciso e eficiente. Conclui-se que o método de área fixa com Parcela Circular foi mais preciso e eficiente para a estimativa das variáveis DAP médio e número de árvores, já que se trata de um método no qual a seleção dos indivíduos é proporcional à área da parcela. O método de Bitterlich, por ser um método que faz a seleção dos indivíduos com probabilidade à área basal, foi mais preciso e eficiente para a estimativa das variáveis área basal e volume total.
\end{abstract}

Palavras-chave: Método de amostragem; precisão e eficiência; Pinus taeda.

\begin{abstract}
Comparison between Bitterlich and fixed area circular plot sampling methods in Pinus taeda L plantation. This study aimed to compare the Bitterlich sampling method to the fixed area circular plot. These two methods were evaluated with respect to the accuracy and efficiency for estimating the variables mean DBH, number of trees, basal area and total volume per hectare. Three conditions for Pinus taeda plantations were selected. They were located in Boa Vista farm, Carambeí County, State of Paraná. Results showed that the fixed area circular plot was more efficient and accurate to estimate the mean DBH and the number of trees per hectare because it is a method where the trees selection is proportional to the plot area, whereas the Bitterlich method was more efficient and accurate to estimate the basal area and the total volume per hectare because the trees selection is related to the basal area.
\end{abstract}

Keywords: Sampling methods; accuracy and efficiency; Pinus taeda.

\section{INTRODUÇÃO}

A preocupação com a mensuração florestal remonta ao início da concepção das ciências florestais na Europa, no final do século XVIII. Prodan et al. (1997) citam Paulsen (1795), Cotta (1804) e Klauprecht (1860) como sendo pioneiros nas questões do desenvolvimento de fórmulas para determinar rendimentos, tabela de volume e fatores de forma. Problemas posteriores que preocuparam esses pioneiros florestais nesta área de estudo, que é a mensuração florestal, foram as funções de crescimento e muito especialmente as técnicas de inventário florestal, que deram origem aos métodos e processos de amostragem no final do século XVIII, mas que só foram aplicados no início do século XX, junto à adoção definitiva das técnicas matemático-estatísticas. 
Atualmente, as empresas trabalham objetivando obter de seus reflorestamentos a produção de madeira para vários usos e, para atender a essas diferentes destinações, têm manejado seus reflorestamentos com enfoque na produtividade, através de desbastes, que se constituem no corte de um percentual de árvores, pelo método seletivo, sistemático ou misto, para a redução da competição e, consequentemente, maior rapidez no crescimento das árvores remanescentes. Dessa forma, para se obter informações básicas para o subsídio do manejo e do planejamento da produção florestal, é necessário o inventário florestal (NAKAJIMA et al., 1998).

Para fins de inventário quali-quantitativo em florestas plantadas, o mais usual ainda é a medição das parcelas em campo, e na literatura encontram-se citados vários métodos de amostragem que podem ser utilizados para obtenção dos dados requeridos. Na Europa, Japão e Estados Unidos, os métodos de amostragem mais utilizados são: método de área fixa com parcela circular (método padrão), método de área fixa com parcela circular concêntrica (método suíço), método de amostragem ponto (método de Bitterlich) e método de amostragem em linha (método de Strand) (NAKAJIMA et al., 1998).

Vasquez (1988) cita que as pesquisas desenvolvidas até então visavam à análise de tamanhos e formas de unidades amostrais aplicadas em diferentes processos de amostragem e baseavam-se nas experiências profissionais, a fim de obter informações pela maximização da precisão e minimização de custos.

Segundo Nakajima et al. (1998), a eficiência dos diferentes métodos de amostragem (tipo, forma e tamanho das parcelas) varia de acordo com o tipo florestal, com as condições da floresta, com as condições da região de estudo e com o tipo do relevo, entre outros aspectos.

Nakajima et al. (1998) citam que a metodologia de amostragem torna-se, assim, um fator decisivo no contexto da amostragem recomendado para uma dada situação, já que a adequação do método de amostragem para as condições da topografia e da floresta possibilitará um aumento da precisão para um mesmo esforço de amostragem, nas estimativas das variáveis de interesse, e uma redução do tempo de execução, o que, consequentemente, refletirá na redução de custos.

Frente aos pontos abordados e citados, fica clara a importância do presente estudo, que visa comparar dois métodos de amostragem: o método de amostragem de área variável, ponto de Bitterlich (PB), e o método de amostragem de área fixa com parcela circular (PC). Além disso, visa escolher adequadamente um desses dois métodos de amostragem, a fim de que possibilite um aumento de precisão para as estimativas das variáveis de interesse e uma redução do tempo de execução da amostragem, reduzindo custos.

O objetivo deste estudo foi comparar a precisão e a eficiência relativa entre os métodos de amostragem ponto de Bitterlich (PB) e amostragem de área fixa com parcela circular (PC) para as estimativas correntes das variáveis diâmetro médio, número de árvores, área basal e volume total, por hectare, em plantios de Pinus taeda L.

O objetivo específico foi testar as seguintes hipóteses:

- Hipótese da nulidade $\left(\mathrm{H}_{0}\right)$ : não existem diferenças estatisticamente significativas entre os métodos de amostragem comparados, tanto para a precisão como para a eficiência relativa, isto é, quaisquer diferenças observadas são devidas exclusivamente aos fatores não controlados ou ao acaso.

- Hipótese alternativa $\left(\mathrm{H}_{1}\right)$ : existem diferenças estatisticamente significativas entre os métodos de amostragem comparados, tanto para a precisão quanto para a eficiência relativa.

\section{REVISÃO DE LITERATURA}

\section{Métodos de amostragem}

Segundo Péllico Netto; Brena (1997), Ara; Musetti; Schneiderman (2003) e Martins (2006), grande parte das pesquisas que envolvem quantificação e qualificação dos recursos florestais são realizadas por meio de estudos dos elementos que compõem uma amostra extraída da população que se pretende analisar.

Péllico Netto; Brena, citados por Sanquetta et al. (2006), citam que método de amostragem significa a abordagem da população referente a uma única unidade de amostra, ou seja, a parcela ou outro tipo de unidade amostral a ser empregada no inventário. Essa abordagem pode ser feita por meio de métodos de área fixa ou de área variável. 
Considerando-se que o inventário florestal é hoje uma atividade que visa obter informações qualitativas e quantitativas dos recursos florestais existentes em uma área pré-especificada, torna-se imprescindível o uso de métodos de amostragem, embora não haja uma classificação completa e diversificada de uso e aplicação em uma dada população (PÉLLICO NETTO; BRENA, 1997).

São vários os métodos de amostragem. No entanto, ao se buscar que um inventário atinja seus objetivos através de uma amostragem de qualidade e representativa da população, convém levar em consideração com bastante clareza dois conceitos fundamentais: precisão e acuracidade.

A precisão refere-se ao tamanho dos desvios da amostra em relação à média estimada, a qual se obtém através da repetição do procedimento de amostragem. Assim, ela será indicada pelo erro padrão da estimativa sem levar em conta o tamanho dos erros não-amostrais.

Para Loetsch et al. (1973), a amostragem é a seleção de parte de um agregado de material para representar o agregado todo.

A informação desejada é obtida com menor custo pela amostragem que pela enumeração total e é mais confiável, porque a observação de apenas parte da população proporciona mais tempo e mais recurso, medindo com mais cuidado, com instrumentos melhores e pessoal mais bem treinado. Com isso, a amostragem oferece precisão desejada, custo mínimo, menos tempo e probabilidade determinada (PÉLLICO NETTO; BRENA, 1997).

Por sua vez, a acuracidade indica o tamanho dos desvios da estimativa amostral em relação à média paramétrica da população (média), inclusive com os erros não-amostrais. Para o inventário florestal, interessa justamente a acuracidade da estimativa amostral. Sendo assim, a precisão é obtida com mais facilidade e, por isso, é mais avaliada.

Método de área fixa com parcelas circulares

Péllico Netto; Brena (1997) dizem que nesse método de amostragem a seleção dos indivíduos é feita proporcionalmente à área da unidade de amostra e, consequentemente, à frequência dos indivíduos que nela ocorrem.

Sterba (1986) relata que a unidade de área fixa é a mais antiga e que nela a seleção dos indivíduos também ocorre com probabilidade proporcional à área, pois todos os indivíduos que se situarem no interior de uma unidade de amostra serão medidos.

Segundo Prodan, citado por Péllico Netto; Brena (1997), as unidades circulares ainda são menos utilizadas no Brasil que as retangulares e quadradas. Porém seu uso vem sendo cada vez mais frequente em inventários florestais. As parcelas circulares ganham eficiência porque, entre todas as formas possíveis, considerando-se a mesma área, são as que possuem menor perímetro e, consequentemente, minimizam o problema de árvores marginais.

Para Sanquetta et al. (2006), as parcelas circulares são mais usuais em inventários de plantações florestais, que requerem unidades menores comparativamente com as demandadas em florestas naturais (geralmente acima de $1.000 \mathrm{~m}^{2}$ ). Exceção é o caso de inventário de regeneração natural.

Segundo Sanquetta et al. (2006), a definição do raio é o aspecto mais importante. Uma parcela circular somente será eficiente se o raio for controlável pelo profissional que está coordenando o inventário no campo. Para os autores, raios grandes, acima de 15 m, não são operacionalmente viáveis e inviabilizam a eficiência do inventário. Em média, a área deve perfazer em torno de 400 a $600 \mathrm{~m}^{2}$.

A parcela circular é um tipo de unidade amostral que mostra tendência de ser cada vez mais adotado em muitas empresas e consultorias na execução de inventários florestais.

Método de área variável com ponto de Bitterlich

Apesar do amplo uso do método de área fixa, métodos alternativos existem e podem se constituir na opção ideal em alguns tipos de inventários, especialmente quando se deseja rapidez e eficiência, como é o caso do método de amostragem de Bitterlich (SANQUETTA et al., 2006).

Conforme Silva (1977), Sterba (1986), Finger (1992) e Prodan et al. (1997), entre outros autores, nesse método, a seleção das árvores que compõem a unidade de amostra é realizada pela comparação do diâmetro da árvore com um ângulo de visada constante.

Farias et al. (2002) mencionam em seu estudo que, devido à simplicidade do procedimento para a obtenção dos dados, a aplicação do método de Bitterlich pode ser de extrema utilidade, principalmente em situações em que se necessita de um diagnóstico rápido do estoque de madeira, entre outras características da floresta. 
Comparando-se o método de Bitterlich, o método de Strand e o método de área fixa nas suas variações de amostragem de parcela circular e de amostragem de parcela circular concêntrica, o método de Bitterlich mostrou-se melhor na estimativa da área basal e volume em povoamento de Pinus (NAKAJIMA et al., 1998).

Precisão e eficiência de unidades amostrais

Oderwald (1981), comparando a precisão para os estimadores de área basal para os métodos de área fixa e variável, concluiu que a precisão varia dependendo do tipo de agregação do plantio, sendo mais preciso o método de área variável para plantios nos quais a distribuição das árvores ocorre aleatoriamente.

Sparcks et al. (2002) compararam estimativas de número de árvores em três plantios no sudeste de Oklahoma (EUA), usando parcelas circulares de tamanho fixo, de tamanho variável e parcelas quadradas. Concluíram que todas as parcelas foram eficientes quanto ao tempo de medição, mas as de área fixa forneceram estimativas mais precisas do número de árvores, independentemente das suas dimensões. As parcelas circulares de tamanho variável e as quadradas tenderam a subestimar o número de árvores real da plantação. Os autores recomendam usar parcelas de área fixa para árvores pequenas (2,5 cm a $11,4 \mathrm{~cm}$ de DAP) e parcelas variáveis para árvores maiores que $11,4 \mathrm{~cm}$ de DAP.

Mauricio et al. (2005), em seus estudos em plantios de Pinus caribaea no oriente da Venezuela, concluíram que, em inventário com parcelas de área fixa, esse método é ligeiramente mais preciso que o de área variável, principalmente quanto à estimativa da variável número de árvores. Os mesmos autores afirmam que a menor precisão do método de área variável é compensada pela maior velocidade de execução e economia nos custos de inventário.

Nakajima et al. (1995) compararam os métodos de amostragem de área fixa, nas variações de parcela circular e circular concêntrica, o de Bitterlich e o de Strand, como um sistema de inventário florestal contínuo para manejo de floresta. Esse estudo foi em Kagoshima, Japão, numa floresta de Cryptomeria japonica D. Don, com 43 anos de idade, misturada com Pinus spp. e diversas folhosas. Na estimativa do número de árvores, área basal e volume por hectare, obtiveram os seguintes resultados: para número de árvores por hectare, o método mais preciso foi o de área fixa com parcela circular, seguido pelo de área fixa com parcela circular concêntrica, e, para a área basal e volume por hectare, o método mais preciso foi o de Bitterlich, seguido pelo de área fixa com parcela circular concêntrica, Strand e de área fixa com parcela circular.

Avery; Burkhart (1950) afirmam que a melhor estrutura de amostragem de um dado problema de estimativa é aquela que estabelece a precisão desejada pelo menor custo. Para eles, isso é obtido com o produto entre o quadrado do erro padrão e o tempo (custo = tempo).

Freese, citado por Péllico Netto; Brena (1997), propõe incluir o custo como um indicador para comparar tamanhos de unidades amostrais, tendo ele importante participação no contexto de avaliação da eficiência das unidades amostrais de diferentes tamanhos. Pode-se usar ainda a combinação dos erros amostrais ou dos coeficientes de variação com os respectivos custos de amostragem.

Péllico Netto; Brena (1997) relatam que, se o objetivo for comparar vários tamanhos simultaneamente, pode-se calcular o inverso dos produtos dos quadrados dos coeficientes de variação pelos respectivos custos e compará-los entre si.

Cesaro et al. (1994), em seu estudo, concluíram que o método de seis árvores mostrou a menor eficiência $(60 \%)$ e o da relascopia $80 \%$, quando comparados com o de área fixa.

Moscovich; Brena; Longhi (1999), em estudo comparativo entre os métodos de área fixa, Strand, Prodan, quadrantes e Bitterlich para as estimativas do número de árvores, área basal e volume com casca por hectare, concluíram que o método mais eficiente foi o de Strand para todas as variáveis estimadas.

\section{MATERIAIS E MÉTODOS}

\section{Caracterização geral da área de estudo}

Para o desenvolvimento desta pesquisa, foram implementadas parcelas em três diferentes talhões com plantios de Pinus taeda L. localizados na Fazenda Boa Vista, de propriedade da Empresa Águia Florestal, sediada no município de Ponta Grossa, PR. Essa fazenda situa-se no município de Carambeí, mesorregião Centro-Oriental do Paraná, na microrregião de Ponta Grossa. Dista cerca de $130 \mathrm{~km}$ de 
Curitiba, sendo $100 \mathrm{~km}$ pela BR 376 até o município de Ponta Grossa e mais 30 pela PR 151. As coordenadas geográficas são $24^{\circ} 04^{\prime} 44^{\prime \prime}$, latitude sul e 5005'49”, longitude oeste.

O clima é classificado como $C f b$ de Köppen, ou seja, um clima subtropical úmido, mesotérmico, com verões frescos, geadas severas, demasiado frequentes e sem estação seca. A precipitação anual média é acima de $1.400 \mathrm{~mm}$ e as chuvas são bem distribuídas durante o ano. A temperatura média anual nos meses mais frios é inferior a $18{ }^{\circ} \mathrm{C}$, enquanto que a média anual dos meses mais quentes é superior a $22^{\circ} \mathrm{C}$ (BOGNOLA et al., 2002).

Os talhões utilizados neste estudo são de Pinus taeda L., sem desbastes, com espaçamentos de $2,00 \mathrm{~m}$ por $2,50 \mathrm{~m}$.

As unidades amostrais foram alocadas em três diferentes talhões, sendo eles:

- talhão PG-009, com área de efetivo plantio de 52,10 ha e idade de 10 anos;

- talhão PG-024, com área de efetivo plantio de 41,52 ha e idade de 7 anos;

- talhão PG-002A e 002B com áreas de efetivo plantio de 10,00 ha e 22,86 ha, respectivamente, que totalizam 32,86 ha e idade de 9 anos.

Os três talhões totalizam 126,48 ha.

Para este estudo, os três diferentes talhões são chamados de condição 1 (talhão PG-009), condição 2 (talhão PG-024) e condição 3 (talhão PG-002A e 002B).

\section{Coleta de dados}

Métodos de medição

Definiu-se o tipo de inventário como sendo o inventário detalhado, e os métodos de amostragem a serem comparados são o método de Bitterlich (PB) e o método de área fixa com parcela circular (PC). O processo de amostragem utilizado foi o aleatório irrestrito, sendo que das unidades amostrais alocadas foram coletadas as seguintes informações: circunferência à altura do peito (CAP), as quais foram convertidas em diâmetro à altura do peito (DAP), algumas alturas e o estado fitossanitário da floresta. Foram alocadas 40 unidades amostrais do método de Bitterlich (PB) e de área fixa com parcela circular (PC). Essas informações forneceram os subsídios básicos para a estimativa de diâmetro médio, número de árvores, área basal e volume total com casca, por hectare.

Instrumentos e materiais utilizados na medição

As medições dos DAPs das árvores inclusas nas parcelas foram feitas pelo método direto, com uso dos seguintes instrumentos e materiais:

- Para o método de amostragem de Bitterlich (PB): relascópio de Bitterlich para a seleção das árvores das parcelas, fita métrica graduada em centímetros para medição dos CAPs, giz para marcação do CAP, estaca para indicação do ponto amostral, ficha de campo, prancheta, lapiseira e borracha.

- Para o método de amostragem de área fixa com parcela circular (PC): estaca, corda com o comprimento do raio da parcela, fita métrica, giz, prancheta, lapiseira e borracha.

- Para os dois métodos foram mensurados o tempo de implantação e a medição, com o auxílio de um cronômetro digital, e, adicionalmente, foram medidas com o auxílio do hipsômetro de Blume-Leiss as alturas de 120 árvores ao acaso presentes nas unidades amostrais dos métodos estudados.

Equipe de campo

A equipe de campo foi composta de três pessoas e mantida em ambos os métodos utilizados. As atribuições de cada integrante em cada método foram:

- Para o método de amostragem por ponto de Bitterlich (PB): um operador de relascópio, um medidor de CAP e um anotador.

- Para o método de amostragem de área fixa com parcela circular (PC): um puxador de corda para verificação do raio da parcela, um medidor de CAP e um anotador.

\section{Métodos de amostragem}

No método de amostragem foram definidos o tamanho e o tipo ou forma das parcelas, de acordo com os métodos de amostragem a serem comparados e as três condições da área em estudo. 
Neste estudo foi comparada a precisão nas estimativas do diâmetro médio, do número de árvores, da área basal e do volume total, por hectare, do método de Bitterlich (PB) e de área fixa com parcela circular (PC).

Método de amostragem por pontos de Bitterlich (PB)

Neste estudo, foi utilizado o fator de área basal dois (FAB 2), porque no uso do FAB 3 ou 4 poucas árvores seriam selecionadas em cada ponto amostral e, consequentemente, diminuiria sua precisão, no caso desta pesquisa.

As fórmulas usadas para as estimativas das variáveis foram sugeridas por Nishizawa, citado por Nakajima (1997).

$$
\begin{array}{r}
d m=\frac{\sum_{i=1}^{n}\left(\frac{d_{i}}{g_{i}}\right)}{\sum_{i=1}^{n}\left(\frac{1}{g_{i}}\right)} \\
N=k * \sum_{i=1}^{n}\left(\frac{1}{g_{i}}\right) \\
G=k * n \\
V=k * \sum_{i=1}^{n}\left(\frac{v_{i}}{g_{i}}\right)
\end{array}
$$

Em que: $d m=$ diâmetro médio $(\mathrm{cm})$;

$N=$ número de árvores por hectare;

$G=$ área basal por hectare $\left(\mathrm{m}^{2} / \mathrm{ha}\right)$;

$V=$ volume por hectare $\left(\mathrm{m}^{3} / \mathrm{ha}\right)$;

$k=$ fator de área basal;

$n=$ número de árvores selecionadas por ponto de amostragem;

$d_{i}=$ diâmetro da árvore "i" selecionada (cm);

$g_{i}=$ área basal da árvore "i”” selecionada $\left(\mathrm{m}^{2}\right)$;

$v_{i}=$ volume da árvore "ij” selecionada $\left(\mathrm{m}^{3}\right)$.

Método de área fixa com parcela circular (PC)

As fórmulas usadas para as estimativas das variáveis foram sugeridas por Nishizawa, citado por Nakajima (1997).

$$
\begin{gathered}
d m=\frac{\sum_{i=1}^{n} d_{i}}{n} \\
N=\frac{n}{a} \\
G=\frac{\sum_{i=1}^{n} g_{i}}{a}
\end{gathered}
$$




$$
V=\frac{\sum_{i=1}^{n} v_{i}}{a}
$$

Em que: $a=$ área da parcela circular (ha). Bitterlich.

Os outros termos são os mesmos descritos para o método de amostragem por pontos de

\section{Número de unidades amostrais}

Para fins deste estudo, foram definidas para as três condições a mesma intensidade amostral, sendo 40 unidades para o método de amostragem por pontos de Bitterlich (PB) e método de amostragem de área fixa com parcela circular (PC).

Como o método de amostragem por pontos de Bitterlich (PB) é um método de área variável, isto é, não possui uma área definida, enquanto que o método de amostragem de área fixa com parcela circular possui um raio e área fixa, uma maneira de transformar os métodos para uma mesma base de comparação, segundo Nakajima (1997), foi ajustar o "tamanho" das parcelas de maneira tal que contenha, em média, uma mesma quantidade de árvores. Assim, o método de área variável (PB) foi utilizado como base e os outros ajustados conforme o número médio de árvores desse método de área variável, conforme utilizado por Nakajima (1997). Sendo assim, na condição 1, 2 e 3, as medidas dos raios são as seguintes: 6,75 m, $5,65 \mathrm{~m}$ e 5,60 m, respectivamente.

\section{Fórmulas estatísticas utilizadas na análise da precisão das variáveis estimadas}

Método de amostragem por ponto de Bitterlich (PB)

Para o método de amostragem por ponto de Bitterlich (PB), as análises estatísticas foram realizadas por meio das seguintes fórmulas, conforme Péllico Netto e Brena, citados por Nakajima (1997).

$$
\begin{gathered}
\bar{x}=\frac{\sum_{i=1}^{n}\left(x_{i}\right)}{n} \\
s_{x}^{2}=\frac{\sum_{i=1}^{n}\left(x_{i}-\bar{x}\right)^{2}}{n-1} \\
s_{x}=\sqrt{s_{x}^{2}} \\
s_{\bar{x}}= \pm \frac{s_{x}}{\sqrt{n}} \\
c v=\frac{s_{x}}{\bar{x}} * 100 \\
E_{r}= \pm \frac{t * s_{\bar{x}}}{\bar{x}} * 100 \\
i c=\bar{x} \pm t * s_{\bar{x}}
\end{gathered}
$$

Em que: $\bar{x}=$ média da variável $\mathrm{x}$;

$x_{i}=$ valor de cada variável $\mathrm{x}$; 
$n$ = número de unidades amostrais;

$s^{2}=$ variância;

$s$ = desvio padrão;

$S_{-}=$erro padrão;

$E r=$ erro de amostragem em porcentagem;

$i c=$ intervalo de confiança.

Método de amostragem com parcela circular (PC)

Para o método de amostragem com parcela circular (PC), as análises estatísticas foram realizadas por meio das fórmulas utilizadas para o método por ponto de Bitterlich (PB), descritas no item anterior.

\section{Estimativa de tempo médio de amostragem}

O tempo médio estimado de amostragem foi obtido através da soma dos tempos de execução da amostra, ou seja, apenas foi cronometrado o tempo para instalação e mensuração de cada parcela. Não foi considerado o tempo de deslocamento, já que os pontos das unidades amostrais foram os mesmos para os métodos de área variável (PB) e de área fixa (PC).

Método da eficiência relativa

Para o cálculo de eficiência relativa foram consideradas todas as variáveis estimadas em cada método de amostragem.

Vasquez (1988) menciona que, para o cálculo da eficiência relativa entre métodos de amostragem, deve-se considerar a precisão, assim como o custo ou o tempo.

Freese, citado por Péllico Netto, Brena (1997), considera os tempos médios de mensuração em cada um dos métodos e os coeficientes de variação obtidos, calculando a eficiência relativa pela seguinte fórmula:

$$
E R=\frac{1}{T_{i} * C V^{2}}
$$

Em que: $E R$ = eficiência relativa;

$T_{i}=$ tempo de medição da unidade amostral " $i$ ";

$C V$ = coeficiente de variação.

Sendo assim, pode-se, com a eficiência relativa, comparar os métodos e mostrar qual obteve melhor desempenho, atendendo ao limite de erro estipulado.

\section{RESULTADOS E DISCUSSÃO}

Os resultados são apresentados para cada condição analisada e para as três condições analisadas simultaneamente.

\section{Condição 1}

São apresentados os modelos hipsométrico e volumétrico, estimativa das variáveis pelos métodos de PB e de área fixa com PC (Tabela 1), análise estatística da variável DAP médio, número de árvores, área basal e volume total (Tabela 2), teste t de Student para as variáveis analisadas (Tabela 3) e a eficiência relativa comparada (Tabela 4).

Melhor modelo hipsométrico e volumétrico

Modelo de Curtis: $\ln h=3,484364+21,30533 *(1 / d)+107,0489 *\left(1 / d^{2}\right)$

Modelo de Schumacher-Hall: $\ln v=-10,60677097+1,957938764 * \ln d+1,190197992 * \ln h$ 
Tabela 1. Estimativa das variáveis DAP médio, número de árvores, área basal, volume total - condição 1.

Table 1. Estimation of variables mean DBH, number of trees, basal area, total volume - condition 1.

\begin{tabular}{lcc}
\hline Método & PB & PC \\
\hline DAP médio $(\mathrm{cm})$ & 17,1 & 16,6 \\
Número médio de árvores $(\mathrm{N} / \mathrm{ha})$ & 1723 & 1619 \\
Área basal média $\left(\mathrm{m}^{2} / \mathrm{ha}\right)$ & 40,15 & 36,94 \\
Volume médio $\left(\mathrm{m}^{3} / \mathrm{ha}\right)$ & 261,33 & 237,24 \\
\hline
\end{tabular}

Tabela 2. Análise estatística da estimativa de DAP médio, número de árvores, área basal e volume total condição 1 .

Table 2. Statatistic analysis of the estimation of mean DBH, number of trees, basal area and total volume - condition 1 .

\begin{tabular}{lcc}
\hline Método & PB & PC \\
\hline DAP médio $(\mathrm{cm})$ & 17,1 & 16,6 \\
Erro padrão $(\mathrm{cm})$ & $\pm 0,20$ & $\pm 0,14$ \\
Erro de amostragem relativo (\%) & $\pm 2,42$ & $\pm 1,71$ \\
\hline Número médio árvores/ha & 1723 & 1619 \\
Erro padrão $(\mathrm{N} / \mathrm{ha})$ & $\pm 48,47$ & $\pm 15,40$ \\
Erro de amostragem relativo (\%) & $\pm 5,69$ & $\pm 1,92$ \\
\hline Área basal média $\left(\mathrm{m}^{2} / \mathrm{ha}\right)$ & 40,15 & 36,94 \\
Erro padrão $\left(\mathrm{m}^{2} / \mathrm{ha}\right)$ & $\pm 0,66$ & $\pm 0,56$ \\
Erro de amostragem relativo (\%) & $\pm 3,31$ & $\pm 3,07$ \\
\hline Volume médio $\left(\mathrm{m}^{3} / \mathrm{ha}\right)$ & 261,33 & 237,24 \\
Erro padrão $\left(\mathrm{m}^{3} / \mathrm{ha}\right)$ & $\pm 4,97$ & $\pm 4,26$ \\
Erro de amostragem relativo (\%) & $\pm 3,84$ & $\pm 3,63$ \\
\hline
\end{tabular}

Tabela 3. Teste t de Student para as variáveis analisadas - condição 1 .

Table 3. Test $t$ of Student for the analysed variables - condition 1 .

\begin{tabular}{lc}
\hline Métodos & PB vs. PC \\
\hline DAP médio $(\mathrm{cm})$ & $2,02^{*}$ \\
N/ha & $2,04^{*}$ \\
G/ha & $3,71^{* *}$ \\
V/ha & $3,68^{* *}$ \\
\hline ** significativo ao nível de 1\% de probabilidade $(\mathrm{p}<0.01) ; *$ significativo ao nível de $5 \%$ de \\
probabilidade $(0.01=<\mathrm{p}<0.05)$; ns: não significativo $(\mathrm{p}>=0.05)$; vs.: versus.
\end{tabular}

$\mathrm{Na}$ estimativa de DAP médio, número de árvores, área basal e volume total, o método de área fixa com PC foi o mais preciso e eficiente.

O teste $\mathrm{t}$ de Student revelou que, para as variáveis estimadas DAP médio e número de árvores, há diferenças significativas ao nível de $5 \%$ de probabilidade entre o método PB vs. PC. Para as variáveis área basal e volume total, o teste t de Student revelou que há diferenças significativas ao nível de $1 \%$ de probabilidade entre o método PB vs. PC.

Tabela 4. Eficiência relativa comparada - condição 1 .

Table 4. Relative compared efficiency - condition 1 .

\begin{tabular}{ccccccccccc}
\hline \multirow{2}{*}{ Método } & Tempo & \multicolumn{2}{c}{ DAP $(\mathbf{c m})$} & \multicolumn{2}{c}{ Árvores/ha } & \multicolumn{2}{c}{ Área basal $\left(\mathbf{m}^{\mathbf{2}} / \mathbf{h a}\right)$} & \multicolumn{2}{c}{ Volume $\left(\mathbf{m}^{\mathbf{3}} / \mathbf{h a}\right)$} \\
\cline { 3 - 12 } & (min.) & cv\% & ER & cv\% & ER & cv\% & ER & cv\% & ER \\
\hline PB & 93,88 & 7,58 & 1,86 & 17,79 & 0,34 & 10,36 & 0,99 & 12,02 & 0,74 \\
PC & 103,36 & 5,34 & 3,39 & 6,01 & 2,67 & 9,60 & 1,05 & 11,35 & 0,75 \\
\hline
\end{tabular}

\section{Condição 2}

São apresentados os modelos hipsométrico e volumétrico, estimativa das variáveis pelos métodos de PB e de área fixa com PC (Tabela 5), análise estatística das variáveis DAP médio, número de 
árvores, área basal e volume total (Tabela 6), teste t de Student para as variáveis analisadas (Tabela 7) e a eficiência relativa comparada (Tabela 8).

Melhor modelo hipsométrico e volumétrico

Modelo de Ogawa: $1 / \mathrm{h}=0,05204485+0,800028 * 1 / d$

Modelo de Schumacher-Hall: $\ln v=-9,678855718+1,961111183 * \ln d+0,825826416 * \ln h$

Tabela 5. Estimativa das variáveis DAP médio, número de árvores, área basal e volume total condição 2.

Table 5. Estimation of variables mean DBH, number of trees, basal area and total volume - condition 2.

\begin{tabular}{lcc}
\hline Método & PB & PC \\
\hline DAP médio $(\mathrm{cm})$ & 15,9 & 16,0 \\
Número médio de árvores (N/ha) & 1768 & 1924 \\
Área basal média $\left(\mathrm{m}^{2} / \mathrm{ha}\right)$ & 35,65 & 40,15 \\
Volume médio $\left(\mathrm{m}^{3} / \mathrm{ha}\right)$ & 169,94 & 191,96 \\
\hline
\end{tabular}

Tabela 6. Análise estatística da estimativa de DAP médio, número de árvores, área basal e volume total condição 2.

Table 6. Statatistic analysis of the estimation of mean DBH, number of trees, basal area and total volume

\begin{tabular}{lcc}
\multicolumn{1}{c}{ - condition 2. } & PB & PC \\
\hline Método & 15,9 & 16,0 \\
\hline DAP médio (cm) & $\pm 0,21$ & $\pm 0,15$ \\
Erro padrão $(\mathrm{cm})$ & $\pm 2,69$ & $\pm 1,91$ \\
Erro de amostragem relativo (\%) & 1768 & 1924 \\
Número médio de árvores (N/ha) & $\pm 49,13$ & $\pm 27,00$ \\
Erro padrão (N/ha) & $\pm 5,62$ & $\pm 2,84$ \\
Erro de amostragem relativo (\%) & 35,65 & 40,15 \\
\hline Área basal média $\left(\mathrm{m}^{2} / \mathrm{ha}\right)$ & $\pm 0,49$ & $\pm 0,79$ \\
Erro padrão $\left(\mathrm{m}^{2} / \mathrm{ha}\right)$ & $\pm 2,78$ & $\pm 4,00$ \\
Erro de amostragem relativo (\%) & 169,94 & 191,96 \\
\hline Volume médio $\left(\mathrm{m}^{3} /\right.$ ha) & $\pm 2,45$ & $\pm 4,22$ \\
Erro padrão $\left(\mathrm{m}^{3} / \mathrm{ha}\right)$ & $\pm 2,91$ & $\pm 4,44$ \\
Erro de amostragem relativo (\%) & &
\end{tabular}

Tabela 7. Teste t de Student para as variáveis analisadas - condição 2.

Table 7. Test $\mathrm{t}$ of Student for the analysed variables - condition 2.

\begin{tabular}{lc}
\hline Método & PB vs. PC \\
\hline DAP médio & $0,38 \mathrm{~ns}$ \\
N/ha & $2,78 * *$ \\
G/ha & $4,82 * *$ \\
V/ha & $4,52 * *$ \\
** significativo ao nível de 1\% de probabilidade $(\mathrm{p}<0.01)$; * significativo ao nível de $5 \%$ de \\
probabilidade $(0.01=<\mathrm{p}<0.05)$; ns: não significativo $(\mathrm{p}>=0.05)$; vs.: versus.
\end{tabular}

Tabela 8. Eficiência relativa comparada - condição 2.

Table 8. Relative compared efficiency - condicion 2.

\begin{tabular}{lccccccccc}
\hline \multirow{2}{*}{ Método } & \multirow{2}{*}{ Tempo (min.) } & \multicolumn{2}{c}{ DAP $(\mathbf{c m})$} & \multicolumn{2}{c}{ Árvores/ha } & \multicolumn{2}{c}{ Área basal $\left(\mathbf{m}^{2} / \mathbf{h a}\right)$} & \multicolumn{2}{c}{ Volume $\left(\mathbf{m}^{\mathbf{3}} / \mathbf{h a}\right)$} \\
\cline { 3 - 11 } & & $\mathbf{c v} \%$ & ER & cv\% & ER & cv\% & ER & cv\% & ER \\
\hline PB & 74,36 & 8,40 & 1,91 & 17,57 & 0,44 & 8,70 & 1,78 & 9,11 & 1,62 \\
PC & 67,12 & 5,98 & 4,17 & 8,87 & 1,89 & 12,51 & 0,95 & 13,89 & 0,77 \\
\hline
\end{tabular}

Na estimativa do DAP médio e número de árvores, o método de área fixa com PC foi o mais preciso e eficiente. Para a estimativa de área basal e volume total, o método PB foi o mais preciso e eficiente. 
$\mathrm{O}$ teste $\mathrm{t}$ de Student revelou que para a variável estimada DAP médio não há diferença significativa entre os métodos PB vc. PC. Para as variáveis número de árvores, área basal e volume total, o teste $\mathrm{t}$ de Student revelou que há diferenças significativas ao nível de $1 \%$ de probabilidade entre o método PB vs. PC.

\section{Condição 3}

São apresentados os modelos hipsométrico e volumétrico, estimativa das variáveis pelos métodos de PB e de área fixa com PC (Tabela 9), análise estatística das variáveis DAP médio, número de árvores, área basal e volume total (Tabela 10), teste t de Student para as variáveis analisadas (Tabela 11) e a eficiência relativa comparada (Tabela 12).

Melhor modelo hipsométrico e volumétrico

Modelo de Trorey: $h=9,81649817-0,06224807 *(d)+0,00778248 *\left(d^{2}\right)$

Modelo de Schumacher-Hall: $\ln v=-9,584241626+1,908064397 * \ln d+0,863903449 * \ln h$

Tabela 9. Estimativa das variáveis DAP médio, número de árvores, área basal e volume total - condição 3.

Table 9. Estimation of variables mean DBH, number of trees, basal area and total volume - condition 3.

\begin{tabular}{lcc}
\hline Métodos & PB & PC \\
\hline DAP médio $(\mathrm{cm})$ & 15,2 & 15,3 \\
Número médio de árvores $(\mathrm{N} / \mathrm{ha})$ & 1764 & 1868 \\
Área basal média $\left(\mathrm{m}^{2} / \mathrm{ha}\right)$ & 33,00 & 35,90 \\
Volume médio $\left(\mathrm{m}^{3} / \mathrm{ha}\right)$ & 176,44 & 192,56 \\
\hline
\end{tabular}

Tabela10. Análise estatística da estimativa de DAP médio, número de árvores, área basal e volume total condição 3.

Table 10. Statatistic analysis of the estimation of mean DBH, number of trees, basal area and total volume - condition 3 .

\begin{tabular}{lcc}
\hline Método & PB & PC \\
\hline DAP médio $(\mathrm{cm})$ & 15,2 & 15,3 \\
Erro padrão $(\mathrm{cm})$ & $\pm 0,14$ & $\pm 0,14$ \\
Erro de amostragem relativo (\%) & $\pm 1,90$ & $\mathbf{1 1 , 8 1}$ \\
\hline Número médio de árvores (N/ha) & 1764 & 1868 \\
Erro padrão (N/ha) & $\pm 44,41$ & $\pm 16,97$ \\
Erro de amostragem relativo (\%) & $\pm 5,09$ & $\mathbf{1 1 , 8 4}$ \\
\hline Área basal média $\left(\mathrm{m}^{2} / \mathrm{ha}\right)$ & 33,00 & 35,90 \\
Erro padrão $\left(\mathrm{m}^{2} / \mathrm{ha}\right)$ & $\pm 0,57$ & $\pm 0,64$ \\
Erro de amostragem relativo $\left(\mathrm{m}^{2} / \mathrm{ha}\right)$ & $\pm \mathbf{3 , 5 1}$ & $\pm 3,61$ \\
\hline Volume médio $\left(\mathrm{m}^{3} / \mathrm{ha}\right)$ & 176,44 & 192,56 \\
Erro padrão $\left(\mathrm{m}^{3} / \mathrm{ha}\right)$ & $\pm 3,05$ & $\pm 3,64$ \\
Erro de amostragem relativo $\left(\mathrm{m}^{3} / \mathrm{ha}\right)$ & $\pm \mathbf{3 , 4 9}$ & $\pm 3,83$ \\
\hline
\end{tabular}

Tabela 11. Teste t de Student para as variáveis analisadas - condição 3.

Table 11. Test $\mathrm{t}$ of Student for the analysed variables - condition 3 .

\begin{tabular}{lc}
\hline Método & PB vs. PC \\
\hline DAP médio & $0,50 \mathrm{~ns}$ \\
N/ha & $2,19^{*}$ \\
G/ha & $3,38^{* *}$ \\
V/ha & $3,39^{* *}$ \\
\hline
\end{tabular}

** significativo ao nível de $1 \%$ de probabilidade (p < 0.01 ); * significativo ao nível de $5 \%$ de probabilidade $(0.01=<\mathrm{p}<0.05)$; ns: não significativo $(\mathrm{p}>=0.05)$; vs.: versus. 
Tabela 12. Eficiência relativa comparada - condição 3.

Table 12. Relative compared efficiency - condition 3.

\begin{tabular}{lccccccccc}
\hline \multirow{2}{*}{ Método } & \multirow{2}{*}{ Tempo (min.) } & \multicolumn{2}{c}{ DAP $(\mathbf{c m})$} & \multicolumn{2}{c}{ Árvores/ha } & \multicolumn{2}{c}{ Área basal $\left(\mathbf{m}^{\mathbf{2}} / \mathbf{h a}\right)$} & \multicolumn{2}{c}{ Volume $\left(\mathbf{m}^{\mathbf{3}} / \mathbf{h a}\right)$} \\
\cline { 3 - 12 } & & $\mathbf{c v} \%$ & ER & cv\% & ER & cv\% & ER & cv\% & ER \\
\hline PB & 71,58 & 5,96 & 3,94 & 15,92 & 0,55 & 10,98 & 1,16 & 10,92 & 1,17 \\
PC & 69,40 & 5,65 & 4,51 & 5,75 & 4,36 & 11,27 & 1,13 & 11,97 & 1,01 \\
\hline
\end{tabular}

Na estimativa de DAP médio e número de árvores, o método de área fixa com PC foi o mais preciso e eficiente. Para a estimativa de área basal e volume total, o método PB foi o mais preciso e eficiente.

O teste $\mathrm{t}$ de Student revelou que para a variável estimada DAP médio não há diferença significativa entre o método PB vs. PC. Para a variável número de árvores, o teste t de Student revelou que há diferenças significativas ao nível de $5 \%$ de probabilidade entre o método de PB vs. PC. Para as variáveis área basal e volume total, o teste t de Student revelou que há diferenças significativas ao nível de $1 \%$ de probabilidade entre o método PB vs. PC.

\section{Comparação simultânea das três condições através do delineamento em blocos ao acaso}

Para esta análise, o delineamento utilizado foi em blocos ao acaso, uma vez que a topografia foi um diferencial, em que cada condição foi considerada como um bloco. Em todas as condições, foi verificada a homocedasticidade ou homogeneidade de variância, seguida de uma análise de variância (ANOVA) e, por fim, caso necessário, o teste de contraste de médias de Tukey. Todas as análises foram efetuadas ao nível de $5 \%$ de probabilidade.

Para estas análises, utilizou-se o software ASSISTAT, versão 7.5 Beta (ASSISTAT, 2008).

Teste de Tukey para o erro de amostragem e eficiência relativa na estimativa de DAP médio, considerando as três condições simultaneamente

As variâncias dos erros de amostragem e eficiência relativa foram homogêneas ao nível de 5\% de probabilidade através do teste de Bartlett.

A análise de variância mostrou que existem diferenças significativas entre pelo menos uma das médias dos tratamentos, tanto para o erro de amostragem quanto para a eficiência relativa, e, sendo assim, para saber quais médias diferem entre si, foi realizado o teste de Tukey (Tabela 13).

Tabela 13. Teste de Tukey para o erro de amostragem e eficiência relativa na estimativa de DAP médio para as três condições simultaneamente.

Table 13. Test of Tukey on the sampling error and relative efficiency for the estimation of mean DBH for all three conditions simultaneously.

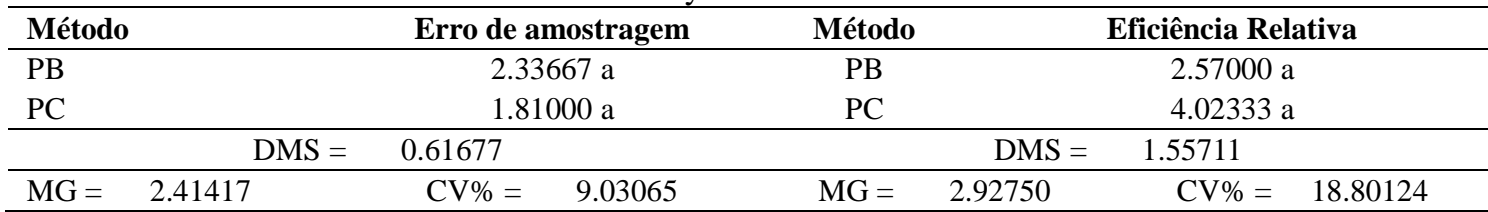

MG: média geral; CV\%: coeficiente de variação em \%; DMS: diferença mínima significativa. As médias seguidas pela mesma letra não diferem estatisticamente entre si. Foi aplicado o teste de Tukey ao nível de 5\% de probabilidade.

Para o erro de amostragem, em relação à estimativa do DAP médio, o teste de Tukey demonstrou que não há diferença estatística entre o método PB vs. PC. Essa análise confirma o PC como o método mais preciso e mais indicado para a estimativa do DAP médio.

Para a eficiência relativa, conforme o teste de Tukey, verifica-se que o PC não difere estatisticamente do $\mathrm{PB}$, sendo o mais eficiente para a estimativa de número de árvores.

Teste de Tukey para o erro de amostragem e eficiência relativa na estimativa de número de árvores, considerando as três condições simultaneamente

As variâncias dos erros de amostragem e eficiência relativa foram homogêneas ao nível de 5\% de probabilidade segundo o teste de Bartlett. 
A análise de variância mostrou que existem diferenças significativas para as médias dos tratamentos, tanto para o erro de amostragem quanto para a eficiência relativa, e, assim, para saber quais médias diferem entre si, foi realizado o teste de Tukey (Tabela 14).

Tabela 14. Teste de Tukey para o erro de amostragem e eficiência relativa na estimativa de número de árvores para as três condições simultaneamente.

Table 14. Test of Tukey on the sampling error and relative efficiency for estimation of number of tree for all three conditions simultaneously.

\begin{tabular}{lccc}
\hline Método & Erro de amostragem & Método & Eficiência relativa \\
\hline PB & $5.46667 \mathrm{a}$ & PB & $0.44333 \mathrm{~b}$ \\
PC & $2.20000 \mathrm{~b}$ & PC & $1.03750 \mathrm{a}$ \\
\hline & $\mathrm{DMS}=0.93239$ & \multicolumn{2}{c}{ DMS $=1.83276$} \\
\hline $\mathrm{MG}=3.57250$ & $\mathrm{CV} \%=13.04683$ & $\mathrm{MG}=1.32917$ & $\mathrm{CV} \%=48.74051$ \\
\hline
\end{tabular}

MG: média geral; CV\%: coeficiente de variação em \%; DMS: diferença mínima significativa. As médias seguidas pela mesma letra não diferem estatisticamente entre si. Foi aplicado o teste de Tukey ao nível de 5\% de probabilidade.

Para o erro de amostragem na estimativa do número de árvores, verifica-se que o PB difere estatisticamente do PC, sendo o PC mais preciso para a estimativa do número de árvores.

Para a eficiência relativa, verifica-se que o PC difere estatisticamente do PB, sendo o mais eficiente para a estimativa de número de árvores.

Para Nakajima (1997), no método de área fixa, a probabilidade de amostragem das árvores é proporcional à frequência. Por isso, na estimativa da variável número de árvores, esse método tende a ser superior ao método de área variável, conforme também constatado nesta pesquisa.

Teste de Tukey para o erro de amostragem e eficiência relativa na estimativa de área basal, considerando as três condições simultaneamente

As variâncias dos erros de amostragem e eficiência relativa foram homogêneas ao nível de 5\% de probabilidade segundo o teste de Bartlett.

A análise de variância para o erro de amostragem na estimativa de área basal mostrou que não há diferenças estatísticas entre as médias dos tratamentos, não necessitando realizar o teste de Tukey. O PB é o método mais preciso na estimativa de área basal, conforme o erro de amostragem apresentado na tabela 15.

A análise de variância da eficiência relativa para a estimativa de área basal mostrou que existem diferenças estatísticas entre pelo menos uma das médias dos tratamentos, e, para verificar quais médias diferem entre si, foi realizado o teste de Tukey (Tabela 15).

Tabela 15. Teste de Tukey para a eficiência relativa na estimativa de área basal para as três condições simultaneamente.

Table 15. Test of Tukey on the sampling error and relative efficiency for estimation of basal area for all three conditions simultaneously.

\begin{tabular}{lccc}
\hline Método & $\begin{array}{c}\text { Erro de } \\
\text { amostragem }\end{array}$ & Método & Eficiência relativa \\
\hline PB & 3.20000 & PB & $1.31000 \mathrm{a}$ \\
PC & 3.56000 & PC & $1.04333 \mathrm{a}$ \\
\hline & - & \multicolumn{2}{c}{$\mathrm{DMS}=0.67177$} \\
\hline- & - & $\mathrm{MG}=0.92917$ & $\mathrm{CV} \%=25.55597$ \\
\hline
\end{tabular}

MG: média geral; CV\%: coeficiente de variação em \%; DMS: diferença mínima significativa. As médias seguidas pela mesma letra não diferem estatisticamente entre si. Foi aplicado o teste de Tukey ao nível de 5\% de probabilidade.

Para a eficiência relativa, o teste revelou que PB vs. PC não diferem estatisticamente entre si.

Segundo Nakajima (1997), pelo fato de no método de ponto de Bitterlich a probabilidade de amostragem das árvores ser proporcional à área basal, na estimativa dessa variável esse método tende a ser superior ao método de área fixa, conforme também constatado nesta pesquisa. 
Teste de Tukey para erro de amostragem e eficiência relativa na estimativa de volume, considerando as três condições simultaneamente

As variâncias dos erros de amostragem e eficiência relativa foram homogêneas ao nível de 5\% de probabilidade segundo o teste de Bartlett.

A análise de variância para o erro de amostragem na estimativa de volume mostrou que não existem diferenças estatísticas, não necessitando realizar o teste de Tukey. O PB é o método mais preciso na estimativa de volume, conforme o erro de amostragem apresentado na tabela 16.

A análise de variância da eficiência relativa para a estimativa de volume mostrou que existem diferenças estatísticas entre as médias dos tratamentos, e, para verificar quais médias diferem entre si, foi realizado o teste de Tukey (Tabela 16).

Tabela 16. Teste de Tukey para a eficiência relativa na estimativa de volume para as três condições simultaneamente.

Table 16. Test of Tukey on the sampling error and relative efficiency for estimation of total volume for all three conditions simultaneously.

\begin{tabular}{lccc}
\hline Método & $\begin{array}{c}\text { Erro de } \\
\text { amostragem }\end{array}$ & Método & Eficiência relativa \\
\hline PB & 3.41333 & PB & $1.17667 \mathrm{a}$ \\
PC & 3.96667 & PC & $0.84333 \mathrm{a}$ \\
\hline- & & \multicolumn{2}{c}{$\mathrm{DMS}=0.59632$} \\
\hline- & - & $\mathrm{MG}=0.79417$ & $\mathrm{CV} \%=26.54173$ \\
\hline MG: média geral; CV\%: coeficiente de variação em \%; DMS: diferença mínima significativa. As \\
médias seguidas pela mesma letra não diferem estatisticamente entre si. Foi aplicado o teste de \\
Tukey ao nível de 5\% de probabilidade.
\end{tabular}

Para a eficiência relativa, o teste revelou que o PB e o PC não diferem estatisticamente entre si. Para Nakajima (1997), o método PB foi o que apresentou o menor erro de amostragem na estimativa da variável volume, também confirmado nesta pesquisa.

\section{CONCLUSÕES E RECOMENDAÇÕES}

Considerando os resultados analisados simultaneamente para as três condições estudadas, aceitase a hipótese da nulidade $\left(\mathrm{H}_{0}\right)$ como verdadeira, isto é, não existem diferenças estatisticamente significativas entre os métodos de amostragem comparados, tanto para a precisão como para a eficiência relativa, ou seja, quaisquer diferenças observadas são devidas exclusivamente aos fatores não controlados ou ao acaso. Dessa forma, conclui-se que:

- Para a estimativa de DAP médio, o método de área fixa com parcela circular (PC) foi mais preciso e eficiente que o de Bitterlich (PB), não havendo diferença estatisticamente significativa entre eles, tanto para o erro de amostragem quanto para a eficiência relativa.

- Para a estimativa de número de árvores, o método de área fixa com parcela circular (PC) foi mais preciso e eficiente que o de Bitterlich (PB), não havendo diferença estatisticamente significativa entre eles, tanto para o erro de amostragem quanto para a eficiência relativa.

- Para a estimativa de área basal, o método de Bitterlich (PB) foi mais preciso e eficiente que o de área fixa com parcela circular, não havendo diferença estatisticamente significativa entre eles, tanto para o erro de amostragem quanto para a eficiência relativa.

- Para a estimativa de volume, o método de Bitterlich (PB) foi mais preciso e eficiente que o de área fixa com parcela circular, não havendo diferença estatisticamente significativa entre eles, tanto para o erro de amostragem quanto para a eficiência relativa.

Recomenda-se a utilização do método de Bitterlich em situações em que o volume é a variável de maior importância, já que os resultados mostraram ser este um método preciso para estimativa direta dessa mesma variável. É um método rápido, por não haver necessidade de mensurar os diâmetros das árvores. Um exemplo de utilização desse método de amostragem seria em inventários de estoques ou précorte para plantações florestais. 
Recomenda-se a utilização da parcela circular em inventários florestais momentâneos, já que os resultados foram precisos. A parcela circular não é utilizada com a finalidade de monitorar o desenvolvimento da floresta, mas, por ser um método de área fixa, tem alta correlação entre medições sequenciais. Sendo assim, pode ser usada com sucesso.

\section{REFERÊNCIAS}

ARA, A. B; MUSETTI, A. V.; SCHNEIDERMAN, B. Introdução à estatística. São Paulo: Instituto Mauá de Tecnologia, 2003. 152 p.

AVERY, T. E.; BURKHART, H. Forest measurements. New York: McGraw-Hill Book Company, 1983. $331 \mathrm{p}$.

BOGNOLA, I.; POTTER, R. O.; CARVALHO, A. P.; FASOLO, P. J.; BHERING, S. B.; MARTORANO, L. G. Caracterização dos solos do município de Carambeí, PR. Boletim de Pesquisa e Desenvolvimento, Rio de Janeiro, p. 75, 2002.

CESARO, A.; ENGEL, O. A.; FINGER, C. O. G.; SCHNEIDER, P. R. Comparação dos métodos de amostragem de área fixa, relascopia e de seis árvores, quanto à eficiência, no inventário florestal de um povoamento de Pinus sp. Ciência Florestal, Santa Maria, v. 4, n. 1, p. 97-108, 1994.

FARIAS, C. A.; SOARES, C. P. B.; SOUZA, A. L; L. H. G. Comparação de métodos de amostragem para análise estrutural de florestas inequiâneas. Revista Árvore, Viçosa, v. 26, n. 5, p. 541-548, 2002.

FINGER, C. A. G. Fundamentos de biometria florestal. Santa Maria: UFSM CEPEF/FATEC, 1993, 263 p.

LOETSCH, F.; ZÖHRER, F.; HALLER, K. E. Forest inventory. 2. ed. Munich: BLV Verlagsgesellschaft, 1973, 469 p.

MAHRER, F.; VOLLENWEIDER, C. National forest inventory. Swiss Federal Institute of Forestry Research, 1983, 26 p.

MARTINS, G. A. Estatística geral e aplicada. 3. ed. São Paulo: Atlas 2006, 421 p.

MAURICIO, J.; VINCENT, L.; MORET, A. Y.; QUEVEDO, A. Comparación entre modalidades de muestreo em plantaciones de Pino Caribe en el oriente de Venezuela. Revista Forestal Venezolana, 49(1), p. 17-25, 2005.

MOSCOVICH, F. A.; BRENA, B. A.; LONGHI, S. J. Comparação de diferentes métodos de amostragem, de área fixa e variável, em uma floresta de Araucaria angustifolia. Ciência Florestal, Santa Maria, v. 9, n. 1, p. 173-191, 1999.

NAKAJIMA, N. Y.; YOSHIDA, S.; IMANAGA, M. Comparison among four ground-survey methods as a continuous forest inventory system for forest management. J. Jpn. For. Soc. v. 77, n. 6, p. 573-580, 1995.

. Comparison of the accuracies of four ground-survey methods used for estimating forest stand values on two occasions. J. For. Plann. v. 2, n. 2, p. 137-144, 1996.

Comparison of change estimation between four ground-survey methods for use in a continuous

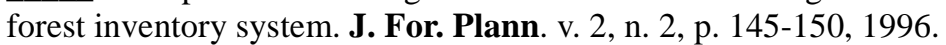

NAKAJIMA, N. Y. Comparison of four ground-survey methods when used as permanent samples in the continuous forest inventory for forest management. Kagoshima University. The United Graduate School of Agricultural Sciences, 1997, 91 p.

NAKAJIMA, N. Y.; KIRCHNER, F. F.; SANQUETTA, C. R. POSONSKI, M. Elaboração de um sistema de amostragem para estimativa de valores correntes e mudança/crescimento em reflorestamento de Pinus. Curitiba: CNPq/UFPR, 1998. 33 p. (CNPq - Pesquisa na modalidade recémdoutor). Projeto concluído. 
ODERWALD, R. G. Comparison of point and plot sampling basal area estimators. Forest Sci. v. 27, n. 1, p. 42-48, 1981.

OGAYA, N. Sobre a influência del tamanho de las parcelas em los errores de muestreo em inventário florestales tropicales. Universidad de Los Andes. Facultad de Ciências Forestales. Centro de Estúdios Forestales de Pós-grado, 1977, p. 153.

PÉLLICO NETTO, S.; BRENA, D. Inventário florestal. Curitiba: Universidade Federal do Paraná, 1997, $316 \mathrm{p}$.

PRODAN, M.; PETERS, R.; COX, F.; REAL, P. Mensura forestal. San José, Costa Rica: IICA, 1997, 586 p.

SANQUETTA, C. R.; WATZLAWICK, L. F.; DALLA CÔRTE, A.; FERNANDES, L. A. V. Inventários florestais: planejamento e execução. Curitiba, 2006, 271 p.

SILVA, L. B. X. Tamanhos e formas de unidades de amostra em amostragem aleatória e sistemática para florestas plantadas de Eucalyptus alba Rewien. 141 p. Dissertação (Mestrado em Engenharia Florestal) - Setor de Ciências Agrárias, Universidade Federal do Paraná, Curitiba, 1977.

SPARKS, J.; MASTERS, R.; PAYTON, M. Comparative evaluation of accuracy and efficiency of six forest sampling methods. Proc. Okla. Acad. Sci, v. 82, p. 49-56, 2002.

STERBA, H. Holzmesslehre. Wien: Inst. f. Forstl. Ertragslehre der Univ. f. Bodenkultur, 1986, 169 p.

UNIVERSIDADE FEDERAL DE CAMPINA GRANDE. ASSISTAT, versão 7.5 (beta). Campina Grande, PB, 2008, http.//www.assistat.com.

VASQUEZ, A. G. Método de amostragem em linhas: desenvolvimento e aplicação em uma floresta implantada com Pinus taeda L. 129 p. Dissertação (Mestrado em Engenharia Florestal) - Setor de Ciências Agrárias, Universidade Federal do Paraná, Curitiba, 1988. 